A novel Ocular Anaesthetic Scoring System, OASS, tool to measure both motor and sensory function following local anaesthesia

Cehajic-Kapetanovic, Jasmina and Bishop, Paul N. and Liyanage, Sidath and King, Trevor and Muldoon, Mark and Wearne, Ian M.

2010

MIMS EPrint: 2010.14

Manchester Institute for Mathematical Sciences

School of Mathematics

The University of Manchester

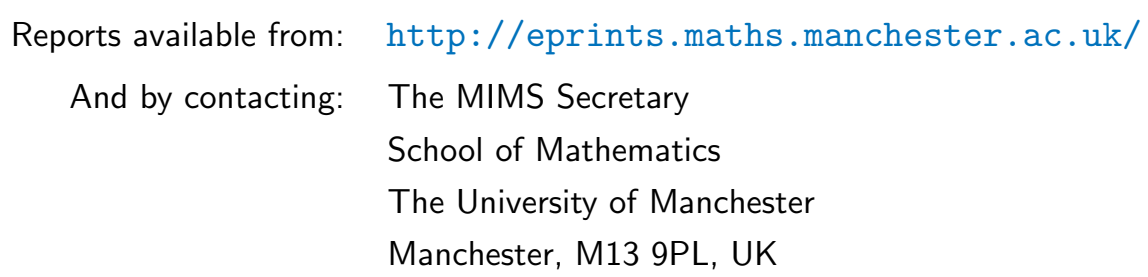

ISSN 1749-9097 


\title{
A novel Ocular Anaesthetic Scoring System, OASS, tool to measure both motor and sensory function following local anaesthesia
}

\author{
J Cehajic-Kapetanovic, ${ }^{1}$ P N Bishop, ${ }^{1}$ S Liyanage, ${ }^{2}$ T King, ${ }^{2}$ M Muldoon, ${ }^{3}$ I M Wearne ${ }^{2}$
}

${ }^{1}$ The University of Manchester, Manchester Academic Health Sciences Centre, Manchester Royal Eye Hospital, Central Manchester Foundation NHS Trust, Manchester, UK;

${ }^{2}$ Department of Ophthalmology and Department of Anaesthetics, Eastbourne District General Hospital, NHS Trust, Eastbourne, UK;

${ }^{3}$ Department of Mathematics \& Statistics, University of Manchester, Manchester, UK

Correspondence to:

Ms J Cehajic-Kapetanovic, Academic Department of Ophthalmology, Manchester Royal Eye Hospital, Oxford Road, Manchester M13 9WH, UK;

jasminac@doctors.org.uk

Accepted 17 July 2009

Published Online First

1 September 2009

\author{
ABSTRACT \\ Background/aims: To devise and evaluate a novel \\ Ocular Anaesthetic Scoring System (OASS) for non- \\ topical local anaesthesia.
}

Methods: In OASS, a score of between 0 (poor) and 14 (excellent) was devised measuring motor (ocular motility, levator and orbicularis function) and sensory functions

(digital spear pressure at limbus and topical anaesthetic sting). 40 patients were studied prospectively to analyse interobserver consistency in OASS. A further 100 patients were collected into four groups receiving either sub-Tenon or peribulbar block with 150 or 300 units of hyaluronidase. Patient satisfaction was determined using the Visual Analogue Pain Scale and lowa Satisfaction with Anaesthesia Scale.

Results: There was no significant difference in OASS scores between two independent observers $(p=0.8910)$. The sub-Tenon approach achieved significantly better OASS scores than the peribulbar approach $(p<0.0004) .300$ units of hyaluronidase gave significantly higher OASS scores in both sub-Tenon $(p<0.0001)$ and peribulbar groups $(p<0.0001)$. Spearman rank correlation showed that OASS correlates significantly with VAPS $(-0.82, p<0.0001)$ and ISAS $(0.70, p<0.0001)$. The median satisfaction score was significant in order of magnitude: sub-Tenon with 300 units of hyaluronidase $>$ sub-Tenon with 150 units of hyaluronidase $>$ peribulbar with 300 units of hyaluronidase $>$ peribulbar with 150 units of hyaluronidase.

Conclusion: OASS is a simple and robust system for assessing and comparing non-topical local anaesthetic techniques. Of the techniques evaluated, a sub-Tenon block with 300 units of hyaluronidase gives the best anaesthesia, analgesia and patient satisfaction results.

Non-topical local anaesthesia is used for many ocular procedures and has a major role in cataract surgery. In the last decade the use of local anaesthesia has increased from $20 \%$ in $1991^{1}$ to $86 \%$ in $1997 .{ }^{2}$ Despite the shift by some surgeons to topical anaesthesia alone, ${ }^{34}$ evidence suggests that pain control during surgery and patient satisfaction with anaesthesia are better achieved with safe non-topical blocks. ${ }^{5-9}$ However, there is currently no consensus as to the optimal approach to non-topical anaesthesia (retrobulbar, peribulbar, sub-Tenon, subconjunctival), and the choice of technique is determined largely by the anaesthetist's or surgeon's preference. As many pharmacological agents are used in different combinations and volumes, and supplementary postadministration techniques vary, different levels of ocular anaesthesia and akinesia are being achieved with each approach. In order to determine the optimal approach, a simple and robust anaesthesia grading system is required.

Currently there is, to our knowledge, no grading system for non-topical anaesthesia that combines measurements of motor and sensory function. Previous studies have measured akinesia alone. ${ }^{1011}$ Alternatively, the efficacy of anaesthesia has been studied through patient satisfaction surveys that relied on qualitative statements by patients and surgeons ${ }^{12}$ or using tools such as the Visual Analogue Pain (VAP) scale ${ }^{73}$ and the Iowa Satisfaction with Anaesthesia Scale (ISAS). ${ }^{14}$

We suggest that a more comprehensive methodology is required to grade ocular anaesthesia that measures both motor and sensory functions. Therefore, the purpose of this study was to develop a novel Ocular Anaesthetic Scoring System (OASS) as a method of grading non-topical anaesthesia using cohorts of patients undergoing cataract surgery. A first study was performed to investigate the effectiveness of the scoring system and to analyse interobserver consistency. A second larger study was then undertaken to compare the effectiveness of different anaesthetic techniques and to determine whether the OASS score correlated with patient satisfaction using the VAP scale and ISAS questionnaire.

\section{MATERIALS AND METHODS}

After obtaining informed consent, we conducted two prospective studies of patients undergoing small incision phacoemulsification surgery. The exclusion criteria were: age $<18$ years, previous intraocular surgery, known allergy to local anaesthetic, intravenous sedation, any coexisting ocular conditions that could affect the scoring system such as ptosis, ocular movement abnormality, reduced corneal sensation, or facial nerve palsy, and inability to understand the information about the study. Data were collected systematically on: age, sex, race, details about anaesthetic technique, duration of surgery, intraoperative difficulties and complications.

All anaesthetic blocks, sub-Tenon or peribulbar, were administered in an anaesthetic room by a senior anaesthetist normally designated to that particular list. The eye to be operated on was painted with povidone iodine, and three drops of topical $1 \%$ proxymethocaine were instilled. During a sub-Tenon block, the patient was asked to look upwards and outwards. Westcott scissors were used to make a small "button-hole" in the conjunctiva and Tenon capsule in the inferonasal 
Table 1 Ocular Anaesthetic Scoring System

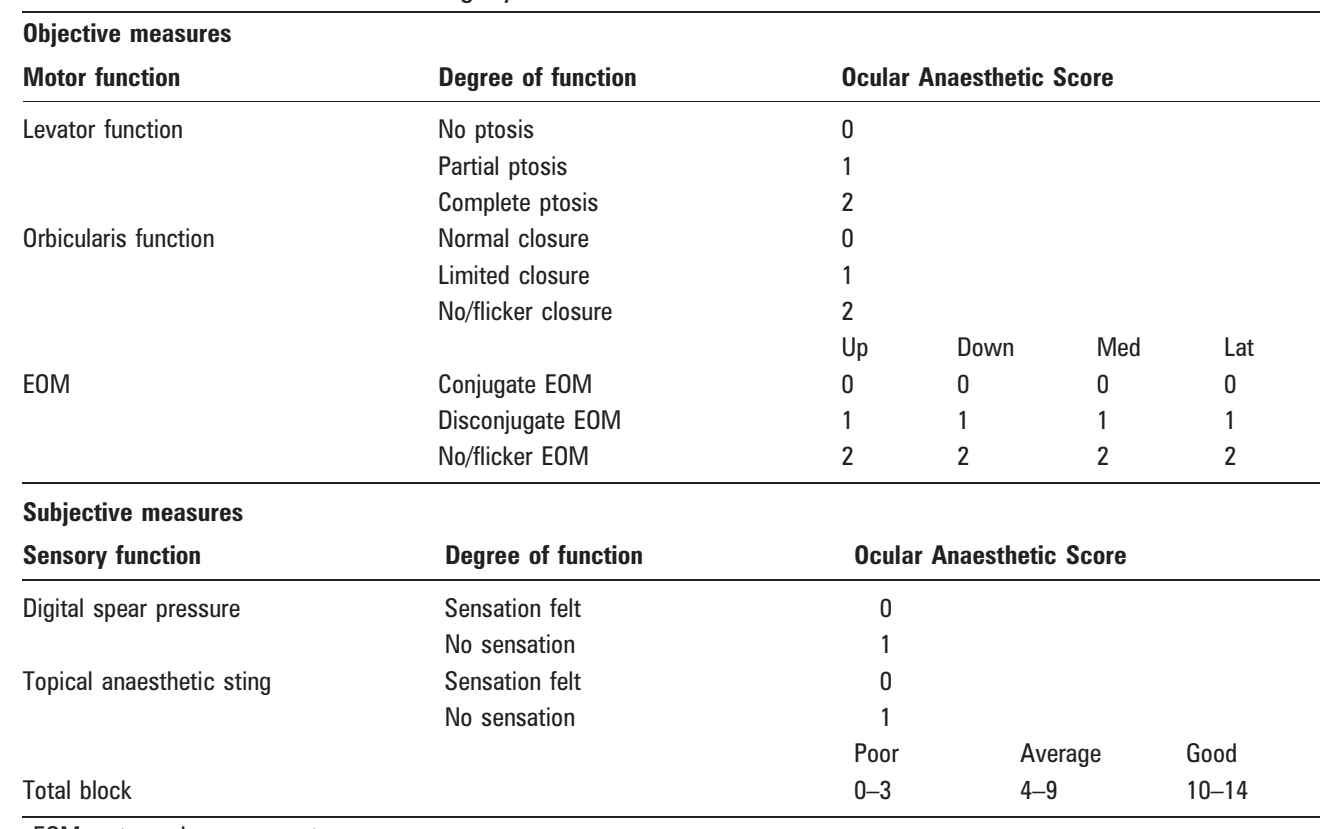

EOM, extraocular movements.

quadrant, $4 \mathrm{~mm}$ from limbus. A blunt dissection was used to create a path in the sub-Tenon space. Conjunctival forceps were used to grip the conjunctiva, and a curved sub-Tenon cannula was then inserted on to bare sclera and glided along the contour of the globe. The anaesthetic mixture was injected slowly in to the posterior sub-Tenon space. For a peribulbar bock, a single injection was given using a $24 \mathrm{G}$ needle at junction of middle and outer third of the lower orbital margin with the needle directed towards the orbital floor.

In order to test the reliability of the OASS scoring system, we conducted a pilot study of 40 patients where we analysed the interobserver consistency. Here, anaesthetists were asked to use their usual preferred "cocktail" and method of administering anaesthesia. The blocks included either the sub-Tenon or peribulbar approach, and they varied in the amount of anaesthetic used (2-8 ml), combination of agents (lidocaine $2 \%$ and bupivocaine $0.75 \%$, lidocaine $2 \%$ alone or lidocaine $2 \%$ with epinephrine 1:10000), amount of hyaluronidase added (0300 units) and postadministration supplementary protocols including the use of weights or ocular massage. The scoring was conducted by the anaesthetist and immediately afterwards by an ophthalmologist (JCK), who was masked to the type of anaesthesia used and the anaesthetist's OASS scoring.

There are three objective measures in OASS of motor function: first the degree of ptosis assessing levator function, second the lid closure assessing orbicularis function and third extraocular eye movements (EOM) assessing akinesia. Grading of each parameter was based on a scoring system between 0 and 2. No ptosis scores zero points, partial one and complete ptosis a maximum of two points. Lid closure was examined by holding the lids gently open and asking the patient to squeeze their eyes shut. Normal closure scores zero points, limited closure one and no or flicker closure two points. Third, eye movements were tested in four positions of gaze: elevation, depression, levo- and dextroversion. Conjugate movements scored zero in each direction, disconjugate movements one and no movement two for each direction. Therefore, zero points were given for normal eye movements, and a total of eight points was given for complete akinesia. There are also two subjective measures in
OASS, digital spear pressure at the limbus and topical anaesthetic sting using a drop of $1 \%$ amethocaine. These are related to sensory function and, if active, can cause unwanted reflex blinking and squeezing of the eyelids. Each was scored zero if sensation was appreciated and one point if there was no sensation. All these scores were added up to give a total score with a maximum of 14 points. Patients were categorised into three groups according to the quality of the block and level of anaesthesia achieved: poor (0-3), average (4-9) and good block (10-14). The Ocular Anaesthetic Scoring System is shown in table 1.

Having devised a scoring system, a further 100 patients were collected to evaluate the effects of different anaesthetic techniques using OASS. In order to avoid bias, all patients undergoing cataract surgery were placed consecutively into one of four treatment groups. The blocks were performed by one of the four anaesthetists (A, B, C or D) who administered the anaesthetic according to the standard techniques described. All patients were injected an anaesthetic "cocktail" consisting of $2 \mathrm{ml}$ of $2 \%$ lignocaine and $2 \mathrm{ml}$ of $0.5 \%$ bupivocaine with the addition of either 150 or 300 units of sodium hyaluronidase via a sub-Tenon or a peribulbar approach. After administration, the eyelid was closed and external pressure applied for $5 \mathrm{~min}$. All

Table 2 Visual Analogue Pain (VAP) scale and lowa Satisfaction with Anaesthesia Scale (ISAS)

\begin{tabular}{|c|c|}
\hline 10-point VAP scale & \\
\hline None $\longrightarrow \mathrm{Pa}$ & ain as bad as it could possibly be \\
\hline B. ISAS questions & C. ISAS responses \\
\hline 1. I was too cold or hot & 1. Agree very much \\
\hline 2. I would want to have the same anaesthetic again & 2. Agree moderately \\
\hline 3. I itched & 3. Agree slightly \\
\hline 4. I felt relaxed & 4. Disagree slightly \\
\hline 5. I felt pain & 5. Disagree moderately \\
\hline 6. I felt safe & 6. Disagree very much \\
\hline 7. I threw up and felt like throwing up & \\
\hline 8. I was satisfied with my anaesthetic care & \\
\hline 9. I felt pain during surgery & \\
\hline 10. I felt good & \\
\hline 11. I hurt & \\
\hline
\end{tabular}


Table 3 Patient demographics

\begin{tabular}{|c|c|c|c|c|c|}
\hline Characteristic & ST-300 (n = 25) & ST-150 $(n=25)$ & PB-300 $(n=25)$ & PB-150 (n = 25) & Significance \\
\hline Age (years) & & & & & NS \\
\hline Mean (SD) & $77.36(11.26)$ & $76.68(8.00)$ & $73.96(9.20)$ & 74.64 (12.77) & \\
\hline Range & 58 to 95 & 60 to 90 & 56 to 89 & 45 to 97 & \\
\hline Sex & & & & & NS \\
\hline Male:female & $11: 14$ & $8: 17$ & $10: 15$ & $12: 13$ & \\
\hline Race & & & & & NS \\
\hline White:Asian & $22: 3$ & $24: 1$ & $25: 0$ & $24: 1$ & \\
\hline Grade of surgeon & & & & & NS \\
\hline Consultant & 13 & 11 & 14 & 15 & \\
\hline Trainee & 11 & 12 & 11 & 9 & \\
\hline Consultant+trainee & 1 & 2 & 0 & 1 & \\
\hline Surgical time (min) & 16.40 & 20.40 & 17.88 & 17.80 & NS \\
\hline $\begin{array}{l}\text { Blocks given by each } \\
\text { anaesthetist }\end{array}$ & & & & & NS \\
\hline A & 5 & 5 & 7 & 8 & \\
\hline B & 9 & 7 & 5 & 4 & \\
\hline C & 6 & 8 & 6 & 5 & \\
\hline D & 5 & 5 & 7 & 8 & \\
\hline
\end{tabular}

patients were blind to the type of anaesthetic given. A single ophthalmologist (JCK) used an OASS before anaesthesia and 5 min after administration. This assessment was done with JCK masked to the type of anaesthesia used. OASS scores were then
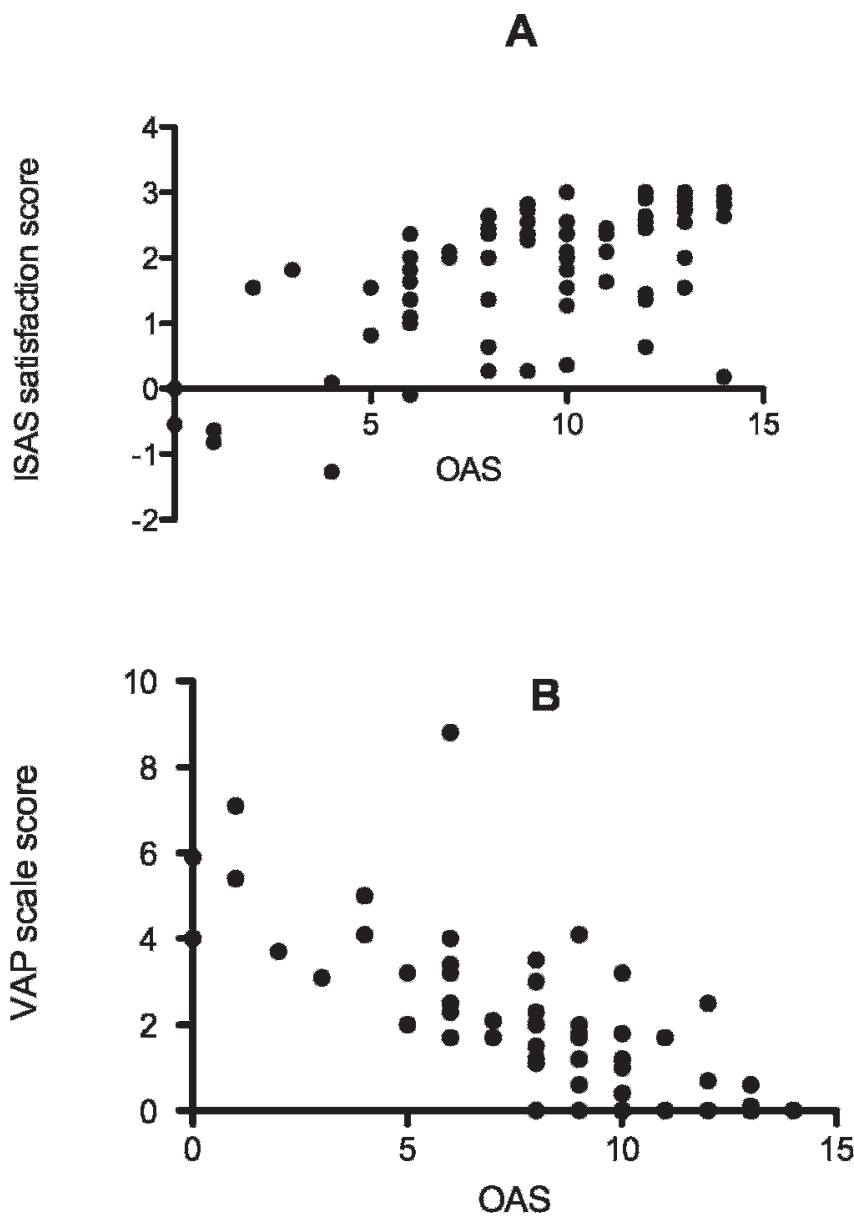

Figure 1 Correlation of Ocular Anaesthetic Score (OAS) with lowa Satisfaction with Anaesthesia Scale (ISAS) score and with Visual Analogue Pain (VAP) scale score. correlated with the patient satisfaction questionnaires (VAP scale and ISAS).

The VAP scale and ISAS are shown in table 2. The 10-point VAP scale is a frequently used method of scoring pain (table 2A). However, pain is only one factor that determines patient satisfaction. A variety of other factors are incorporated into ISAS. This is a well-established tool devised to reliably score patient satisfaction with respect to their anaesthesia care. It is a written questionnaire consisting of 11 positive and negative statements, which are weighted to remove bias. Patients respond to these statements (table $2 \mathrm{~B}$ ) by placing a mark along a six choice vertical response column for each statement (table 2C). A totally satisfied patient receives a score of +3 , whereas a totally dissatisfied patient receives a score of -3 . The mean of their responses to the 11 statements results in a single number between -3 and +3 representing a quantitative measure of a patient's satisfaction with their anaesthesia care. Patients were issued the questionnaire soon after their recovery from theatre. Specific instructions were given to patients stating that their responses were anonymous and confidential, and would not influence their care in any way. None of the investigators were in contact with patients during the survey, and no one helped them fill in the form.

\section{Statistical analysis}

Quantitative data were entered into Prism 5 Statistical Package software for analysis. In the case of significance, a nonparametric test was used, based on the assumptions that the main outcome measures, OASS, VAPS and ISAS scores, are not normally distributed. Correlations between quantitative variables were estimated with the Spearman coefficient. p Values less than 0.05 were considered statistically significant.

\section{RESULTS}

The study of interobserver consistency showed that there was no statistically significant difference in OASS scores between two independent observers, an ophthalmologist and anaesthetist (two-tailed Mann-Whitney U test, $p=0.8910$ ). In fact, the same scores were obtained in $80 \%$ of cases, and they were within one point in all cases. There was a considerable range in the level of anaesthesia achieved, with scores ranging from 0 to 


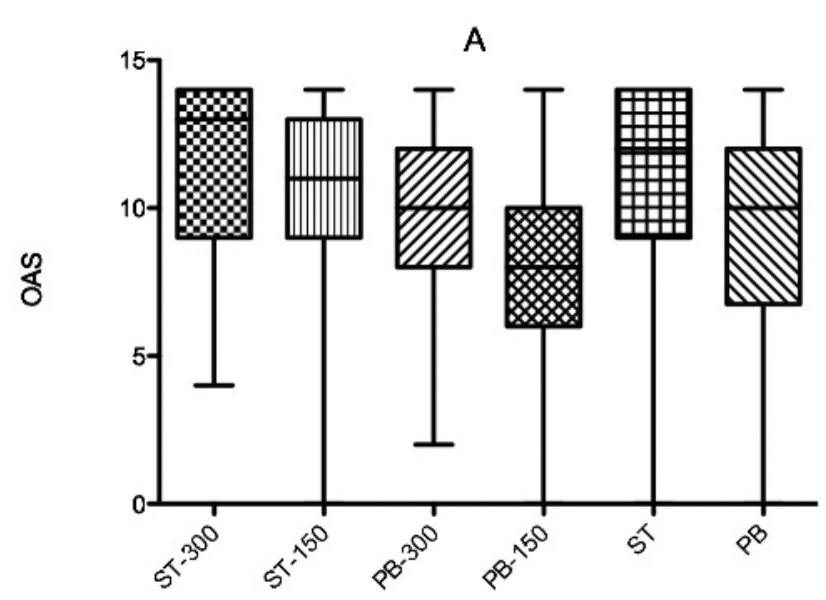

Anaesthetic Block

B

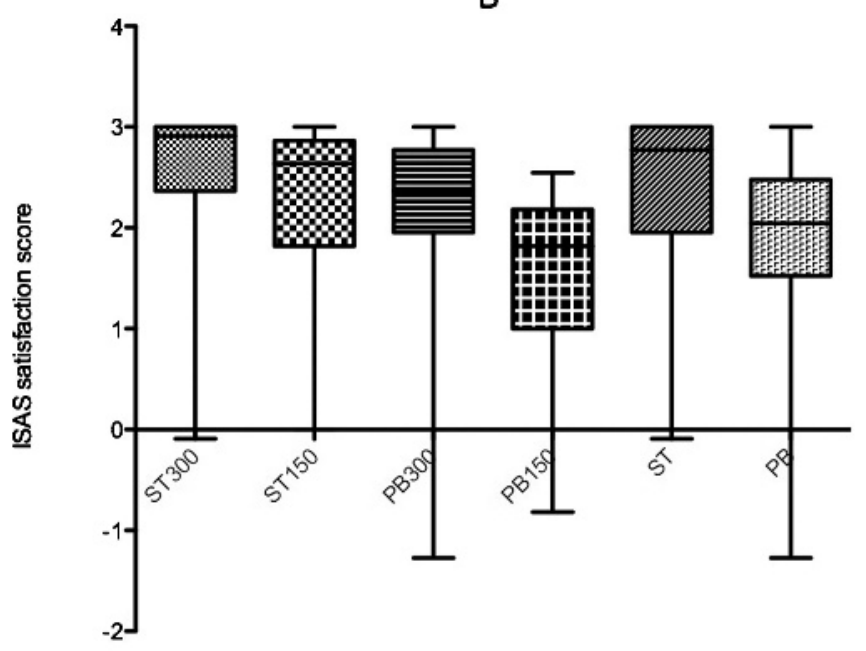

Anaesthetic Block

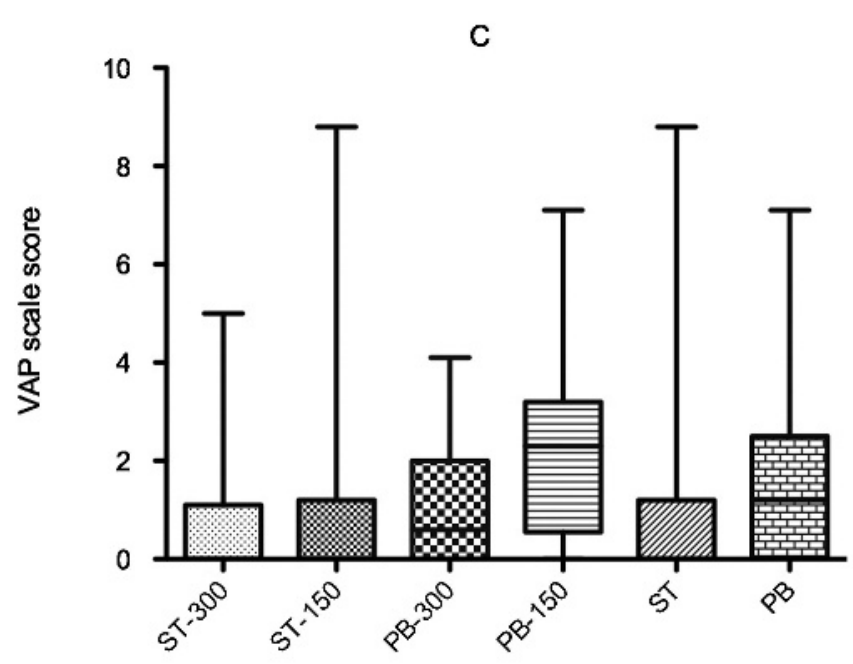

Anaesthetic Block

Figure 2 Box and whisker plots of (A) Ocular Anaesthetic Score (OAS), (B) ISAS satisfaction score and (C) Visual Analogue Pain (VAP) scale score with different anaesthetic blocks. ST-300, sub-Tenon anaesthesia with 300 units of hyaluronidase; ST-150, sub-Tenon anaesthesia with 150 units of hyaluronidase; PB-300, peribulbar anaesthesia with 300 units of hyaluronidase; PB-150, peribulbar anaesthesia with 150 units of hyaluronidase; ST, combined data form sub-Tenon anaesthesia with either 300 or 150 units of hyaluronidase; PB, combined data from peribulbar anaesthesia with either 300 or 150 units of hyaluronidase.
14, depending upon the anaesthetic "cocktail" and technique used.

The effectiveness of anaesthetic techniques and patient satisfaction with anaesthesia was determined in our follow-on study of a further 100 patients. The analysis of patient demographics is shown in table 3 . No statistically significant differences between the groups were identifiable (one-way ANOVA) which might have influenced the scoring.

There were no adverse effects due to anaesthesia, and there were no intraoperative complications. Seven patients required "top-up" topical (4) or non-topical (3) anaesthesia prior to completion of the surgery. Twenty-five patients received a subTenon (ST) block with 300 units of hyaluronidase (ST-300), 25 an ST block with 150 units of hyaluronidase (ST-150), 25 a peribulbar block (PB) with 300 units of hyaluronidase and 25 a PB block with 150 units of hyaluronidase (PB-150). All patients had an OASS of zero prior to administration of anaesthesia. Using OASS, the ST approach achieved significantly better scores than the PB approach (two-tailed Mann-Whitney U test, $\mathrm{p}<0.0004$ ). Higher levels of hyaluronidase (300 units) were associated with significantly higher scores in both ST and PB groups (two-tailed Mann-Whitney $U$ test, $p<0.0001$ ). Spearman rank correlation showed a significant positive correlation $(0.70, p<0.0001)$ between the OASS scores and the ISAS questionnaire (Figure 1A). A significant negative correlation was demonstrated between the OASS scores and the VAP scale $(-0.82, \mathrm{p}<0.0001)$ (fig $1 \mathrm{~B})$. Non-parametric analysis of variance (Kruskal-Wallis statistic) showed a significant difference between the groups in terms of OASS $(p<0.0015)$. The median scores were 13 (IOR 9 to 14) for ST-300, 11 (IOR 9 to 13) for ST-150, 10 (IOR 8 to 12) for PB-300 and 8 (IOR 6 to 10) for the PB-150 group. There was also a significant difference in patient satisfaction between all the above groups using both VAP and ISAS scales (Figure 2). The median VAP score was zero (no pain felt) for both ST-300 (IOR 0 to 1.1) and ST-150 (IOR 0 to 1.2), whereas for the peribulbar groups the median score was 0.6 (IOR 0 to 2) for PB-300 and 2.3 (IOR 0.6 to 3.2) for PB-150. Interestingly, $20 / 25$ patients $(80 \%)$ in $\mathrm{PB}-150$ group reported feeling pain compared with only $7 / 25(28 \%)$ in the ST-300 group. Of all patients who experienced pain 49/100, 76\% reported pain at the end of surgery, $18 \%$ at the beginning and $6 \%$ in the middle of the surgery. The median ISAS scores were 2.91 (IOR 2.36 to 3.00) for ST-300, 2.64 (IOR 1.81 to 2.86) for ST-150, 2.36 (IOR 1.96 to 2.77) for PB-300 and 1.81 (IOR 1.00 to 2.18) for $\mathrm{PB}-150$ group. The median satisfaction score was higher in ST (2.77, IOR 1.96 to 3.00) compared with the PB (2.05, IOR 1.52 to 2.48$)$ group and in the high-300 units (2.59, IOR 2.09 to 3.00 ) compared with the low-150 units (2.00, IOR 1.43 to 2.64) hyaluronidase group. Overall, the median satisfaction score was significant in order of magnitude: subTenon with 300 units of hyaluronidase>sub-Tenon with 150 units of hyaluronidase > peribulbar with 300 units of hyaluronidase>peribulbar with 150 units of hyaluronidase.

\section{DISCUSSION}

We have developed a novel OASS that systematically quantifies in a combined single score both motor and sensory functions in ocular anaesthesia. We found the scoring system to be simple, quick and reproducible. Having evaluated OASS, we have shown it to be an excellent tool in comparing anaesthesia between different techniques. Overall, we found that the ST approach achieved significantly better OASS scores than the PB approach, thus demonstrating that the choice of technique matters. Furthermore, higher doses of hyaluronidase were 
associated with significantly higher OASS scores in both the ST and $\mathrm{PB}$ groups. In addition, we found that our objective OASS scores correlated well with a patient's experience of pain and overall satisfaction in ocular anaesthesia. In particular, patient satisfaction scores followed the same pattern as the OASS scores with ST being significantly superior to $\mathrm{PB}$ and the high dose of hyaluronidase being significantly better than the low dose. Possible hypotheses as to why ST is superior to PB are that there is less discomfort during the administration due to the use of a blunt cannula rather than a sharp needle in $\mathrm{PB}$, better distribution of anaesthetic and less increase in intraocular pressure which, when high, can cause discomfort. Furthermore, the difference in anaesthesia achieved between ST and PB blocks may reflect the fact that orbital blocks commonly require more than $4 \mathrm{ml}$ of anaesthetic, and many anaesthetists will provide a second injection via the caruncle.

Previous studies that have compared ST with PB anaesthesia have not combined objective grading of motor and sensory functions or correlated their objective scores to patients' subjective experience of overall satisfaction. Briggs et $a{ }^{13},^{13}$ for example, compared the efficacy of the two techniques based on patient discomfort alone, during injection of anaesthetic and during surgery, using a 10 -point VAP scale. Parkar et a $1^{15}$ compared efficacy using different parameters that were analysed separately: a 12-point scale to grade akinesia, a 4-point scale to grade each of chemosis, subconjunctival haemorrhage and positive pressure during surgery and a 4-point VAP scale to grade pain during administration of anaesthesia, during surgery and $4 \mathrm{~h}$ after surgery. Some parameters scored higher for ST, some higher for $\mathrm{PB}$, whereas for some there was no difference between the two techniques. There was no combined score to grade the overall efficacy of either anaesthesia.

Hyaluronidase aids the spread of the anaesthetic through the extracellular matrix by depolymerising hyaluronic acid and other glycosaminoglycans. There are variable results in the current literature regarding the effect of hyaluronidase on ocular anaesthesia. Alwitry et al ${ }^{16}$ reported that hyaluronidase (150 units) facilitates the onset of ocular anaesthesia, whereas Moharib et $a l^{17}$ showed no difference in the time of onset of akinesia using 75 units of hyaluronidase. Aslam et a $1^{11}$ demonstrated an improvement in ocular and lid akinesia with the addition of 75 units of hyaluronidase. Our study shows that a group of patients who received 300 units of hyaluronidase had better OASS scores compared with a group that received 150 units. We further demonstrated an improvement in patient's subjective experience of pain and satisfaction with higher doses of hyaluronidase.

There were no adverse effects in our study from ST or PB anaesthesia in combination with hyaluronidase, and there were no complications during surgery. However, previous studies do report a better safety profile with ST compared with PB blocks which can cause respiratory arrest, ${ }^{18}$ generalised epilepsy and severe hypotension ${ }^{19}$ and globe rupture. ${ }^{20}$

In conclusion, we have devised OASS to help objectively quantify anaesthesia during ocular surgery. It allows methods for non-topical blocks to be compared and optimised as well as improving communication between anaesthetist and surgeon, thereby allowing for a more patient-centred approach and reducing the risk of dissatisfaction. ST with a higher dose of hyaluronidase gave the best results, whereas $\mathrm{PB}$ with a low level of hyaluronidase gave the worst results in terms of anaesthesia, analgesia and patient satisfaction. All groups were comparable in terms of complications and safety profiles.

Acknowledgements: We acknowledge support from Manchester NIHR Biomedical Research Centre.

Competing interests: None.

Patient consent: Obtained.

Provenance and peer review: Not commissioned; externally peer reviewed.

\section{REFERENCES}

1. Hodgkins PR, Luff AJ, Morrell AJ, et al. Current practice of cataract extraction and anaesthesia. Br J Ophthalmol 1992;76:323-6.

2. Desai $\mathbf{P}$, Reidy A, Minassian DC. Profile of patients presenting for cataract surgery in the UK: national data collection. Br J Ophthalmol 1999;83:893-6.

3. Kershner RM. Topical anaesthesia for small incision self-sealing cataract surgery. A prospective evaluation of the first 100 patients. J Cataract Refract Surg 1993;19:290-2.

4. Grabow HB. Topical anaesthesia for cataract surgery. Eur J Implant Refract Surg 1993:5:20-4.

5. Friedman DS, Bass EB, Lubomski LH. Synthesis of the literature on the effectiveness of regional anaesthesia for cataract surgery. Ophthalmology 2001;108:519-29.

6. Srinivasan S, Fern Al, Selveraj S, et al. Randomised double-blind clinical tria comparing topical and sub-Tenon's anaesthesia in routine cataract surgery. Br J Anaesth 2004;93:683-6.

7. Ruschen H, Celaschi D, Bunce C, et al. Randomised controlled trial of sub-Tenon's block versus topical anaesthesia for cataract surgery: a comparison of patient satisfaction. Br J Ophthalmol 2005;89:291-3.

8. Roman SJ, Chong Sit DA, Boureau CM, et al. Sub-Tenon's anaesthesia: an efficient and safe technique. Br J Ophthalmol 1997;81:673-6.

9. Davison M, Padroni S, Bunce C, et al. Sub-Tenon's anaesthesia versus topical anaesthesia for cataract surgery. Cochrane Database Syst Rev 2007;(18):CD006291.

10. Brahma AK, Pemberton CJ, Ayeko M, et al. Single medial injection peribulbar anaesthesia using prilocaine. Anaesthesia 1994;49:1003-5.

11. Aslam S, Sarker SJ, Tran-Dang M, et al. Effect of hyaluronidase on ocular motility and eyelid function in sub-Tenon's anaesthesia: randomised controlled trial. Eye 2006;20:579-82.

12. Raman SV, Barry JS, Murjaneh S, et al. Comparison of $4 \%$ articaine and $0.5 \%$ levobupivacaine/2\% lidocaine mixture for sub-Tenon's anaesthesia in phacoemulsification cataract surgery: a randomised controlled trial. $\mathrm{Br} J$ Ophthalmol 2008;92:496-9.

13. Briggs MC, Beck SA, Esakowitz L. Sub-Tenon's versus peribulbar anaesthesia for cataract surgery. Eye 1997;11:639-43.

14. Dexter F, Aker J, Wright WA. Development of a measure of patient satisfaction with monitored anaesthesia care: The lowa Satisfaction with Anaesthaesia Scale. Anesthesiology 1997;87:865-73.

15. Parkar T, Gogate P, Deshpande M, et al. Comparison of subtenon anaesthesia with peribulbar anaesthesia for manual small incision cataract surgery. Indian J Ophthalmol 2005; 53:255-9.

16. Alwitry A, Chaudhary S, Gopee K, et al. Effect of hyaluronidase on ocular motility in sub-Tenon's anaesthesia: randomized controlled trial. J Cataract Refract Surg 2002;28:1420-3.

17. Moharib MM, Mitra S, Rizvi SG. Effect of alkalinization and/or hyaluronidase adjuvancy on a local anaesthetic mixture for sub-Tenon's ophthalmic block. Acta Anaesthesiol Scand 2002;46:599-602.

18. Singer SB, Preston R, Hodge WG. Respiratory arrest following peribulbar anesthesia for cataract surgery: case report and review of the literature. Can J Ophthalmol 1997;32:450-4.

19. Calenda E, Olle P, Muraine $\mathbf{M}$, et al. Peribulbar anesthesia and sub-Tenon injection for vitreoretinal surgery: 300 cases. Acta Ophthalmol Scand 2000;78:196-9.

20. Rathi V, Basti S, Gupta S. Globe rupture during digital massage after peribulbar anesthesia. J Cataract Refract Surg 1997;23:297-9. 